\title{
Rectal Pain, CTCAE 5.0
}

National Cancer Institute

\section{Source}

National Cancer Institute. Rectal Pain, CT CAE 5.0. NCI Thesaurus. Code C146631.

A disorder characterized by a sensation of marked discomfort in the rectal region. 